\title{
Energy band alignment at the nanoscale
}

Cite as: Appl. Phys. Lett. 110, 051603 (2017); https://doi.org/10.1063/1.4975644

Submitted: 07 December 2016 • Accepted: 24 January 2017 • Published Online: 03 February 2017

(iD) Jonas Deuermeier, Elvira Fortunato, Rodrigo Martins, et al.

\section{ARTICLES YOU MAY BE INTERESTED IN}

Highly conductive grain boundaries in copper oxide thin films

Journal of Applied Physics 119, 235303 (2016); https://doi.org/10.1063/1.4954002

Visualization of nanocrystalline $\mathrm{CuO}$ in the grain boundaries of $\mathrm{Cu}_{2} \mathrm{O}$ thin films and effect on band bending and film resistivity

APL Materials 6, 096103 (2018); https://doi.org/10.1063/1.5042046

Reactive magnetron sputtering of $\mathrm{Cu}_{2} \mathrm{O}$ : Dependence on oxygen pressure and interface formation with indium tin oxide

Journal of Applied Physics 109, 113704 (2011); https://doi.org/10.1063/1.3592981

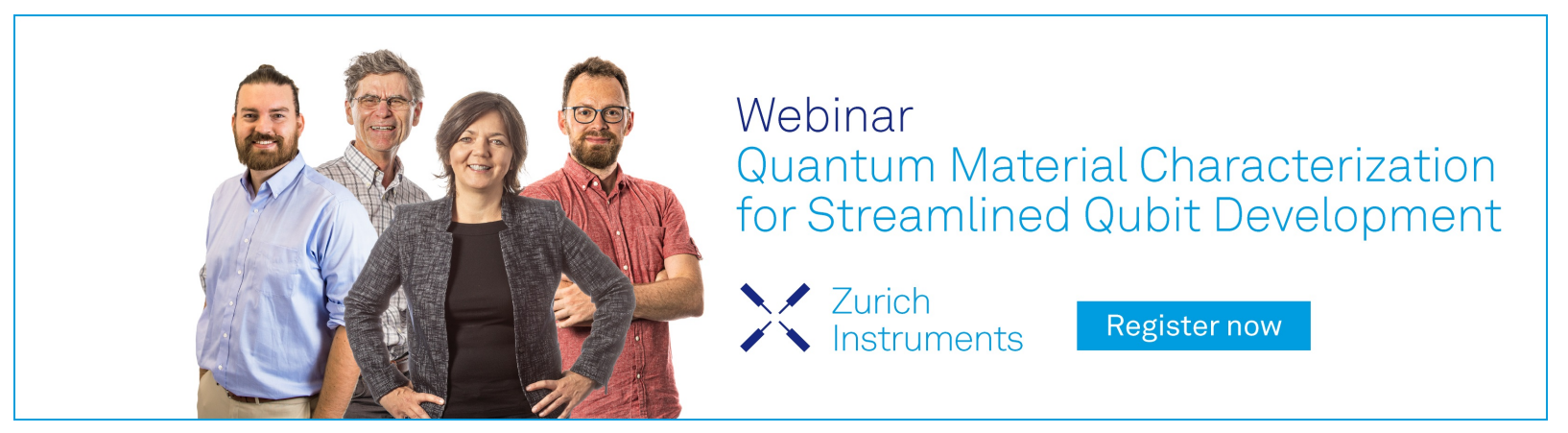




\title{
Energy band alignment at the nanoscale
}

\author{
Jonas Deuermeier, ${ }^{1,2, a)}$ Elvira Fortunato, ${ }^{1}$ Rodrigo Martins, ${ }^{1}$ and Andreas Klein ${ }^{2, b)}$ \\ ${ }^{1}$ i3N/CENIMAT, Department of Materials Science, Faculty of Science and Technology, Universidade NOVA \\ de Lisboa and CEMOP/UNINOVA, Campus de Caparica, 2829-516 Caparica, Portugal \\ ${ }^{2}$ Department of Materials and Earth Sciences, Technische Universität Darmstadt, \\ Jovanka-Bontschits-Straße 2, D-64287 Darmstadt, Germany
}

(Received 7 December 2016; accepted 24 January 2017; published online 3 February 2017)

The energy band alignments at interfaces often determine the electrical functionality of a device. Along with the size reduction into the nanoscale, functional coatings become thinner than a nanometer. With the traditional analysis of the energy band alignment by in situ photoelectron spectroscopy, a critical film thickness is needed to determine the valence band offset. By making use of the Auger parameter, it becomes possible to determine the energy band alignment to coatings, which are only a few Ångström thin. This is demonstrated with experimental data of $\mathrm{Cu}_{2} \mathrm{O}$ on different kinds of substrate materials. Published by AIP Publishing.

[http://dx.doi.org/10.1063/1.4975644]

The measurement of the energy band alignment between a film and a substrate material is possible by a step-wise procedure of alternating film deposition and in situ photoelectron spectroscopy. In the absence of chemical shifts and charging artifacts, the valence band offset is typically extracted at film thicknesses where a parallel shift of binding energies of the substrate and film emissions is observed. ${ }^{1}$ The binding energy shift is a result of the charge exchange at the interface, since this creates charged states in the layers or at the interface. When the photoemission occurs in close proximity to the substrate (for example, in a very thin film), the substrate may affect the screening of the photoholes in the film material. This is mainly related to the influence of the substrate on the polarizability of electrons around the emission site in the film. ${ }^{2}$ As a consequence, the binding energies of substrate and film emissions do not shift in parallel. In order to determine the valence band offset, a minimum film thickness is required, so that the substrate has no influence on the photoholes screening in the film.

The Auger parameter $\alpha^{\prime}$ is sensitive to a change in polarizability, since the emission of a photoelectron creates a single excited state, whereas the Auger emission (AE) involves two ionized states. ${ }^{3}$ Therefore, tabulated values of $\alpha^{\prime}$ are a widely used tool to obtain chemical state information from a material. ${ }^{4}$ Other than by a change in the oxidation state, a shift in $\alpha^{\prime}$ can occur due to different coordinations of a metal cation (as it is the case of $\mathrm{Al}^{3+}$ in $\mathrm{Al}_{2} \mathrm{O}_{3}$, deposited on $\mathrm{SiO}_{2}$ ). ${ }^{5}$ Furthermore, bridging oxygen between two different cations affects $\alpha^{\prime}{ }^{6}$ The changes in the coordination number and bridging oxygen at an interface are rather short-ranged phenomena, since they depend on the bonding to the nearest neighboring ion/atom. An eventually different dielectric constant of the two materials forming the interface also affects the polarizability. The latter contribution extends further into the photoemitting material than the next neighboring environment. ${ }^{6,7}$

An evaluation of the usefulness of the Auger parameter in order to correct the binding energies of film emissions for

\footnotetext{
${ }^{\text {a)} E l e c t r o n i c ~ m a i l: ~ j . d e u e r m e i e r @ c a m p u s . f c t . u n l . p t ~}$

b)Electronic mail: aklein@surface.tu-darmstadt.de
}

the determination of the energy band alignment to the substrate is lacking. In this work, a set of experiments on different substrate materials is presented, in which the Auger parameter of copper oxide $\mathrm{Cu}_{2} \mathrm{O}$ is used to remove the extraatomic relaxation from the binding energy shifts towards the interface to the substrate. In this way, it is shown that such a correction of the measured binding energies reduces the critical film thickness for a determination of the valence band offset to a few Ångström.

Sample preparation for in situ X-ray photoelectron spectroscopy (XPS) was done at the DArmstadt Integrated SYstem for MATerial research (DAISY-MAT), a cluster tool which provides UHV transfer between deposition and characterization chambers. ${ }^{8} \mathrm{Cu}_{2} \mathrm{O}$ thin films were grown by reactive radio frequency (rf) magnetron sputtering without substrate heating. A 2 in. metallic copper target of $99.999 \%$ purity purchased from Lesker was sputtered at $25 \mathrm{~W}\left(2.53 \mathrm{~W} \mathrm{in.}{ }^{2}\right)$ and $0.5 \mathrm{~Pa}$ total pressure. The stoichiometry of $\mathrm{Cu}_{2} \mathrm{O}$ was optimized by adjusting the gas flow ratio of oxygen with respect to the total gas flow in the range of $3.7 \%-5.0 \%$. The details on indicators for $\mathrm{Cu}_{2} \mathrm{O}$ stoichiometry by in situ XPS can be found in the respective literature. ${ }^{9-11}$ The substrate materials which are analyzed in this work are $\mathrm{Al}_{2} \mathrm{O}_{3}$ by atomic layer deposition (ALD) as well as $\mathrm{Bi}_{2} \mathrm{O}_{3}$ and indium-tin oxide (ITO) by reactive rf magnetron sputtering. $\mathrm{Al}_{2} \mathrm{O}_{3}$ as substrate material was tested in two variants: First, a $250 \mathrm{~nm}$ thick multi-layer dielectric of alternating layers of $\mathrm{Al}_{2} \mathrm{O}_{3}$ and $\mathrm{TiO}_{2}$ by ALD on ITOcoated glass was used, which is referred to as ATO and was provided by Planar Systems (now Beneq). The surface of this substrate consisted of $25 \mathrm{~nm}$ pure $\mathrm{Al}_{2} \mathrm{O}_{3}$. Second, $25 \mathrm{~nm}$ of $\mathrm{Al}_{2} \mathrm{O}_{3}$ was deposited on a commercial ITO-coated glass substrate in a custom-made ALD chamber. This substrate is referred to as plain $\mathrm{Al}_{2} \mathrm{O}_{3}$ in the remainder of this article. The parameters for a deposition at $200{ }^{\circ} \mathrm{C}$ are described elsewhere. ${ }^{12}$ The deposition of $45 \mathrm{~nm} \mathrm{Bi}_{2} \mathrm{O}_{3}$ by reactive rf magnetron sputtering was performed onto a commercial ITO-coated glass without intentional substrate heating according to the process described in a previous publication. ${ }^{11}$ The ITO substrate was deposited on glass without intentional substrate heating according to the process described in previous work. ${ }^{10}$ 
XPS was performed in a Physical Electronics PHI 5700 multi-technique surface analysis unit, using monochromatic $\mathrm{Al} \mathrm{K} \alpha$ radiation with an energy of $h \nu=1486.6 \mathrm{eV}$, an emission angle of $45^{\circ}$, and a pass energy of $5.85 \mathrm{eV}$, resulting in an overall energy resolution of less than $0.4 \mathrm{eV}$.

The binding energy $E_{\mathrm{B}}$ is obtained by photoelectron spectroscopy as a positive value with respect to the Fermi energy $E_{\mathrm{F}}$ at $E_{\mathrm{B}}=0$. The magnitude of $E_{\mathrm{B}}$ is a function of the electron density at the emitting ion or atom. ${ }^{13}$ The binding energy would be purely determined by the initial state if the electron density remained "frozen" upon the creation of a photohole. In reality, the electron density around the emitting ion/atom screens the photohole due to relaxation processes, which constitute the final state. ${ }^{2}$ The binding energy can be expressed as the difference between the initial state energy $\epsilon$ and the final state energy $R .{ }^{13}$ Shifts in binding energy of a photoelectron core level $\Delta E_{\mathrm{B}}(\mathrm{PE})$ with respect to an isolated state can have several reasons, which are generally categorized as initial state effects $\Delta \epsilon$ and final state effects $\Delta R .^{3,13,14}$

It is useful for the here presented analysis to distinguish two effects separately, which contribute to a binding energy shift and are included in Eq. (1). Hollinger used $\Delta E_{\mathrm{F}}$ to account for changes in Fermi energy at an interface between two materials. ${ }^{15}$ If thermodynamic equilibrium is assumed at a junction, it is of course the energy bands which change with respect to a constant Fermi energy. However, as the Fermi energy is used as reference energy in XPS, a parallel shift of energy bands may technically be expressed as a change in Fermi energy with the opposite sign. Iwata and Ishizaka, furthermore, added the term $e \Delta \Phi$ in order to account for the effects of charging, where $e$ is the elementary charge. ${ }^{16}$ Charging due to photoemission of electrons always increases the binding energy, which is why its sign should be positive

$$
\Delta E_{\mathrm{B}}(\mathrm{PE})=\Delta \epsilon-\Delta R-\Delta E_{\mathrm{F}}+e \Delta \Phi .
$$

As mentioned earlier, only when the binding energy shift is exclusively due to the change in Fermi energy $\Delta E_{\mathrm{F}}$, the binding energies of substrate and film emissions shift in parallel and the valence band offset can be determined. This requires the knowledge of $\Delta R$, besides a stable chemistry of the materials at the junction $(\Delta \epsilon=0)$ and the absence of charging artifacts $(e \Delta \Phi=0)$. In order to determine the change in relaxation energy $\Delta R$, the Auger parameter $\alpha^{\prime}$ can be used according to ${ }^{3,13}$

$$
\Delta R=\frac{\Delta \alpha^{\prime}}{2} .
$$

The relaxation phenomena, which determine $\Delta R$, can be local (i.e., intra-atomic) or extra-atomic. Since $\mathrm{Cu}(\mathrm{I})$ does not undergo a local relaxation process, ${ }^{6}$ only extra-atomic relaxation is considered for the remainder of this article.

The Auger parameter is defined as the sum of the binding energy of the photoelectron $E_{\mathrm{B}}(\mathrm{PE})$ and the kinetic energy of the Auger electron $E_{\mathrm{kin}}(\mathrm{AE})$, which is equivalent to the difference between $E_{\mathrm{B}}(\mathrm{PE})$ and the binding energy at which the Auger emission appears in the spectrum $E_{\mathrm{B}}(\mathrm{AE})=h \nu-E_{\mathrm{kin}}(\mathrm{AE})^{13}$

$$
\alpha^{\prime}=E_{\mathrm{B}}(\mathrm{PE})+E_{\mathrm{kin}}(\mathrm{AE})=E_{\mathrm{B}}(\mathrm{PE})+h \nu-E_{\mathrm{B}}(\mathrm{AE}) .
$$

With the changes in polarizability due to the proximity to an interface, a change in $\alpha^{\prime}$ can be gradually observed towards low film thicknesses. ${ }^{17,18}$ Figure 1 shows a schematic example of how $\alpha^{\prime}$ manifests in the binding energy shifts of $\mathrm{Cu}_{2} \mathrm{O}$ close to the interface to a substrate material. Below a critical film thickness, $\Delta R$ is not equal to zero and can be calculated according to Equation (2). This allows to obtain the photoelectron binding energy shift due to the band bending $\Delta E_{\mathrm{B}}^{\mathrm{bb}}(\mathrm{PE})$, which is free of extra-atomic relaxation caused by the vicinity of the substrate and is equivalent to a change in Fermi energy position due to the contact formation between the two materials

$$
\Delta E_{\mathrm{B}}^{\mathrm{bb}}(\mathrm{PE})=\Delta E_{\mathrm{B}}(\mathrm{PE})+\Delta R=-\Delta E_{\mathrm{F}} .
$$

The binding energy shifts of different core levels of the substrate and the film material were evaluated at each film thickness. Both for the substrate and film material, the shift is uniform for all core levels and can thus be represented by the respective valence band maximum. The results are displayed with respect to the film thickness in Figure 2. The core levels used for the calculation are indicated in the legend below the plots. The binding energy shift caused by the band bending $\Delta E_{\mathrm{B}}^{\mathrm{bb}}(\mathrm{PE})$ is calculated using the $\mathrm{Cu} 2 \mathrm{p}$ emission and the Cu LMM Auger emission and represented in red. LMM refers to the orbitals in X-ray level notation, which are involved in the Auger emission, more precisely $\mathrm{L}_{3} \mathrm{M}_{4,5} \mathrm{M}_{4,5}$. For each experiment, the valence band offset $\Delta E_{\mathrm{VB}}$ is determined using both the non-corrected binding energy shifts (black vertical lines and numbers) and the binding energy shift of $\mathrm{Cu} 2 \mathrm{p}$ caused by the band bending (red vertical lines and numbers).

Figure 2(a) shows the results for the interface between $\mathrm{Cu}_{2} \mathrm{O}$ and the ATO substrate. Charging effects due to the limited substrate conductance appear at intermediate thicknesses. However, the contribution $e \Delta \Phi$ is the same for both

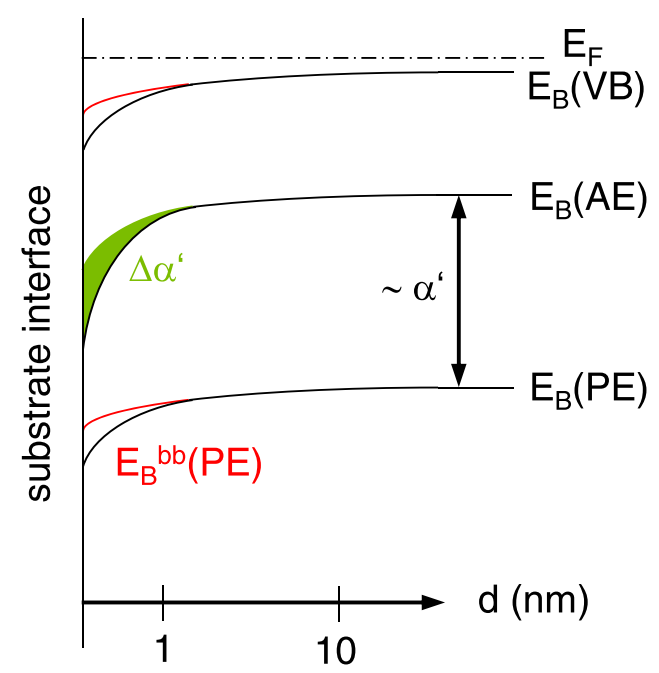

FIG. 1. Schematic representation of extra-atomic relaxation in $\mathrm{Cu}_{2} \mathrm{O}$ at the interface to the substrate depending on the film thickness $d$. The measured binding energies $E_{\mathrm{B}}$ of the photoemission (PE) and the Auger emission (AE) as well as the valence band maximum (VB) are represented as black lines with respect to the Fermi energy $E_{\mathrm{F}}$. The change in the Auger parameter $\Delta \alpha^{\prime}$ is shown in green. When $\Delta R=\Delta \alpha^{\prime} / 2$ is added to the photoelectron binding energy, the red lines $\Delta E_{\mathrm{B}}^{\mathrm{bb}}(\mathrm{PE})$ are obtained, which applies to all photoelectron emissions. 

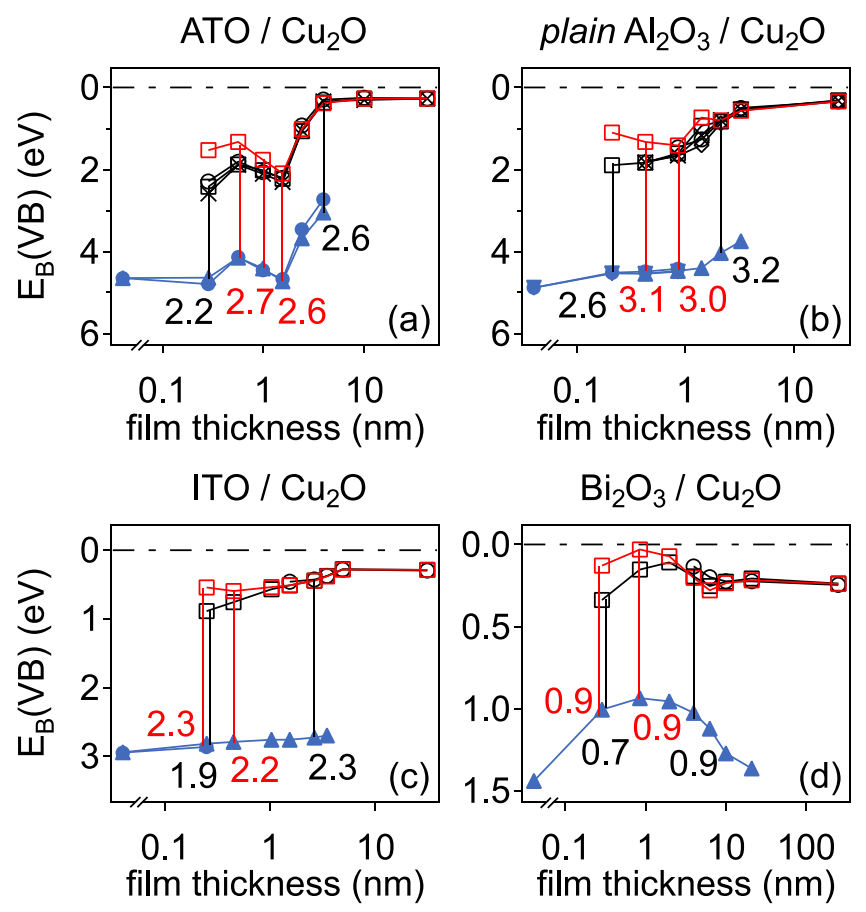

substrate emissions

\begin{tabular}{|c|}
\hline $\begin{array}{l}{\left[\mathrm{E}_{\mathrm{B}}(\mathrm{VB})\right]_{\text {bulk }}+\Delta \mathrm{E}_{\mathrm{B}}(\mathrm{PE})} \\
\multimap \mathrm{Al} 2 \mathrm{~s}, \mathrm{Bi} 4 \mathrm{f}, \ln 3 \mathrm{~d} \\
\multimap \mathrm{Al} 2 \mathrm{p} \multimap \mathrm{O} 1 \mathrm{~s}\end{array}$ \\
\hline $\begin{array}{l}\text { Cu } 2 p-\text { Cu 3s } \\
\times \text { Cu } 3 p-0 \text { O 1s } \\
{\left[E_{B}(V B)\right]_{\text {bulk }}+\Delta E_{B}^{b b}(P E):} \\
\square \text { Cu } 2 p+\Delta R\end{array}$ \\
\hline
\end{tabular}

film emissions

FIG. 2. The binding energy of the valence band maxima of $\mathrm{Cu}_{2} \mathrm{O}$ films and of the different substrates are shown with respect to film thickness. In each representation, the binding energy increases from $E_{\mathrm{F}}=0 \mathrm{eV}$ towards the bottom. The evaluation of extra-atomic relaxation in $\mathrm{Cu}_{2} \mathrm{O}$ is included as $\Delta E_{\mathrm{B}}^{\mathrm{bb}}(\mathrm{PE})$ of the $\mathrm{Cu} 2 \mathrm{p}$ emission.

the substrate and the film emissions. Since artifacts due to charging were absent both in the measurement of the clean substrate and the film at final thickness, the evaluation of the valence band offset is not hindered. The respective value is found to range between 2.2 and $2.6 \mathrm{eV}$, when the measured $\mathrm{Cu} 2 \mathrm{p}$ binding energy is used for the calculation. Using $\Delta E_{\mathrm{B}}^{\mathrm{bb}}(\mathrm{PE})$ leads to a constant value of $\Delta E_{\mathrm{VB}}=2.6 \mathrm{eV}$ between 0.6 and $4 \mathrm{~nm}$ film thickness.

The interface between plain $\mathrm{Al}_{2} \mathrm{O}_{3}$ and $\mathrm{Cu}_{2} \mathrm{O}$ shown in Figure 2(b) reveals no sign of charging artifacts. This is probably due to a presumably better substrate conductance. Although all core levels of substrate and film individually show consistent shifts, there is some irregularity in the core level binding energies of the film material at $1.8 \mathrm{~nm}$. The origin of this is not clear. $\Delta E_{\mathrm{VB}}$ ranges from 2.6 to $3.2 \mathrm{eV}$ when calculated from the measured binding energy shifts. The value corrected for the extra-atomic relaxation due to the substrate is $3.1 \pm 0.1 \mathrm{eV}$ between 0.4 and $4 \mathrm{~nm}$ film thickness.

The data presented in Figure 2(c) show $\mathrm{Cu}_{2} \mathrm{O}$ on ITO. The core level binding energies are taken from previous work. ${ }^{10}$ In the original publication, the Auger parameter has not been evaluated. Removing the additional extra-atomic relaxation caused by the substrate at a low film thickness allows us to determine a valence band offset of $\Delta E_{\mathrm{VB}}$ $=2.2-2.3 \mathrm{eV}$ at film thicknesses between 0.3 and $3.5 \mathrm{~nm}$.

Figure 2(d) shows the interface between $\mathrm{Bi}_{2} \mathrm{O}_{3}$ and $\mathrm{Cu}_{2} \mathrm{O}$. The consistency of the $\Delta E_{\mathrm{VB}}$ quantification with respect to the film thickness can be improved, when the binding energy shift caused by the band bending $\Delta E_{\mathrm{B}}^{\mathrm{bb}}(\mathrm{PE})$ is used. Then, a value of $0.9 \mathrm{eV}$ is observed in a film thickness range between 0.3 and $6.3 \mathrm{~nm}$.

A comparison of the different substrate materials in terms of their influence on $\Delta R$ with increasing thickness is given in Figure 3. It is observed that $\mathrm{Al}_{2} \mathrm{O}_{3}$ as substrate has a more severe effect on extra-atomic relaxation at low thicknesses of $\mathrm{Cu}_{2} \mathrm{O}$ than $\mathrm{Bi}_{2} \mathrm{O}_{3}$ and ITO, the latter showing no significant difference between each other. Since the changes in polarizability due to different dielectric constants of the substrate $\epsilon_{\mathrm{S}}$ and film materials $\epsilon_{\mathrm{f}}$ are effective beyond the nearest neighbor environment, the corresponding polarization energy $U(d)$ shall be calculated here with respect to the film thickness $d^{7,17}$

$$
U(d) \approx \frac{\pi e^{2}}{8 d}\left(\frac{1}{\epsilon_{\mathrm{f}}}-\frac{1}{\epsilon_{\mathrm{s}}}\right) .
$$

$\mathrm{Bi}_{2} \mathrm{O}_{3}$ has a dielectric constant of $40,{ }^{19}$ compared to 9 of $\mathrm{Al}_{2} \mathrm{O}_{3},{ }^{20}$ which is rather similar to $\mathrm{Cu}_{2} \mathrm{O} .{ }^{21}$ Although ITO is not degenerately doped when deposited at room temperature, its dielectric constant is assumed to be infinite, similar to a metal. The resulting values are compared to $\Delta R$ in Figure 3.

Since $U(d)$ is positive whereas $\Delta R$ is negative, the different dielectric constants of substrate and film materials are not the main cause for the observed negative $\Delta R$. However, since the effect is undoubtedly present, it may simply counteract a more prominent relaxation phenomenon at the interface. Such a compensation of a negative $\Delta R$ would be stronger for $\mathrm{Cu}_{2} \mathrm{O}$ on ITO and $\mathrm{Bi}_{2} \mathrm{O}_{3}$ than for $\mathrm{Cu}_{2} \mathrm{O}$ on $\mathrm{Al}_{2} \mathrm{O}_{3}$. This interpretation is supported by a study in which $\mathrm{Cu}_{2} \mathrm{O}$ was deposited on $\mathrm{SiO}_{2}$ and $\mathrm{ZrO}_{2} \cdot{ }^{22} \mathrm{SiO}_{2}$ has a lower dielectric constant than $\mathrm{Cu}_{2} \mathrm{O}$, whereas $\mathrm{ZrO}_{2}$ a higher one. ${ }^{20} \Delta \alpha^{\prime}$ towards low film coverages was found to be negative in both cases and to decrease more strongly on the less polarizable substrate $\left(\mathrm{SiO}_{2}\right)$ than on the one with higher polarizability $\left(\mathrm{ZrO}_{2}\right)$. This means that, for the results presented in this contribution, the change in polarizability by the substrate is predominantly related to the

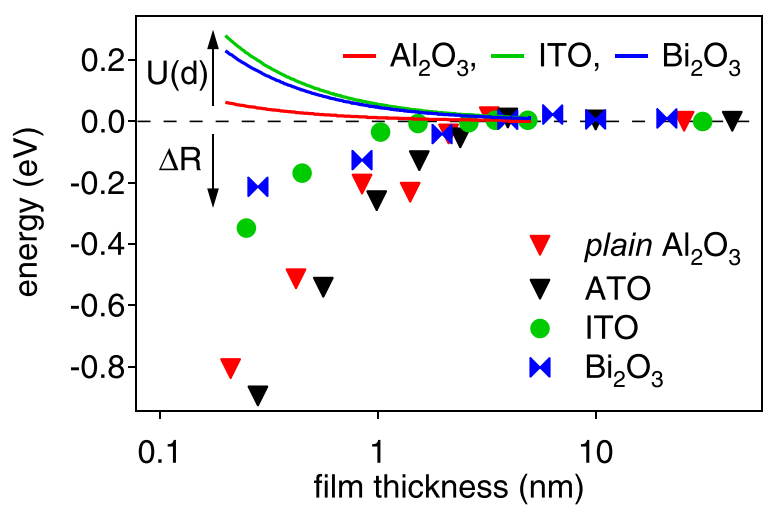

FIG. 3. Extra-atomic relaxation $\Delta R$ and polarization energy $U(d)$ with respect to $\mathrm{Cu}_{2} \mathrm{O}$ thickness $d$ on the different substrate materials. 
chemical bonds between the substrate and film material and the resultant changes of cation coordination in the film. ${ }^{23}$

In summary, it could be shown that it is possible to determine the energy band alignment by photoelectron spectroscopy between two materials, of which at least one is thinner than a nanometer. This was achieved by correcting the binding energy shift of the film emissions for the extraatomic relaxation $\Delta R$ due to the vicinity of the substrate interface. The latter was quantified using the change in the Auger parameter. Since extra-atomic relaxation is an inherent effect of the photoemission process, it is concluded that the valence band offset between two materials is fully developed at a film thickness of a few Angström. Using the described procedure, the valence band offsets between $\mathrm{Cu}_{2} \mathrm{O}$ and $\mathrm{Al}_{2} \mathrm{O}_{3}$ by $\mathrm{ALD}(2.6 \mathrm{eV})$ as well as $\mathrm{Cu}_{2} \mathrm{O}$ and $\mathrm{Bi}_{2} \mathrm{O}_{3}$ $(0.9 \mathrm{eV})$ were determined.

This work was funded by FEDER funds through the COMPETE 2020 program and national funds through FCTPortuguese Foundation for Science and Technology under the Project No. POCI-01-0145-FEDER-007688, Reference No. UID/CTM/50025 as well as SFRH/BD/77103/2011, UID/ CTM/50025/2013, PEst-C/CTM/LA0025/2013-14 and EXCL/ CTM-NAN/0201/2012. Furthermore, it has been supported by the German Science Foundation within the collaborative research center SFB 595 (Electrical Fatigue of Functional Material).

${ }^{1}$ J. R. Waldrop, W. Grant, S. P. Kowalczyk, and E. A. Kraut, J. Vac. Sci. Technol., A 3, 835 (1985).
${ }^{2}$ W. Egelhoff, Surf. Sci. Rep. 6, 253 (1987).

${ }^{3}$ C. Wagner and A. Joshi, J. Electron Spectrosc. Relat. Phenom. 47, 283 (1988).

${ }^{4}$ J. F. Moulder, W. F. Stickle, P. E. Sobol, and K. D. Bomben, Handbook of X-ray Photoelectron Spectroscopy (Physical Electronics, Inc., 1995).

${ }^{5}$ R. Reiche, F. Yubero, J. Espinós, and A. González-Elipe, Surf. Sci. 457, 199 (2000).

${ }^{6}$ A. R. González-Elipe and F. Yubero, Spectroscopic characterization of oxideloxide interfaces, Handbook of Surfaces and Interfaces of Materials, 2nd ed., edited by H. S. Nalwa (Academic Press, San Diego, 2001).

${ }^{7}$ J. G. Kirkwood, J. Chem. Phys. 2, 351 (1934).

${ }^{8}$ A. Klein, J. Am. Ceram. Soc. 96, 331 (2013).

${ }^{9}$ P. E. Larson, J. Electron Spectrosc. Relat. Phenom. 4, 213 (1974).

${ }^{10}$ J. Deuermeier, J. Gassmann, J. Brötz, and A. Klein, J. Appl. Phys. 109, 113704 (2011).

${ }^{11}$ J. Deuermeier, H. F. Wardenga, J. Morasch, S. Siol, S. Nandy, T. Calmeiro, R. Martins, A. Klein, and E. Fortunato, J. Appl. Phys. 119, 235303 (2016).

${ }^{12}$ T. J. M. Bayer, A. Wachau, A. Fuchs, J. Deuermeier, and A. Klein, Chem. Mater. 24, 4503 (2012).

${ }^{13}$ C. D. Wagner, Faraday Discuss. Chem. Soc. 60, 291 (1975).

${ }^{14}$ T. D. Thomas, J. Electron Spectrosc. Relat. Phenom. 20, 117 (1980).

${ }^{15}$ G. Hollinger, Appl. Surf. Sci. 8, 318 (1981).

${ }^{16} \mathrm{~S}$. Iwata and A. Ishizaka, J. Appl. Phys. 79, 6653 (1996).

${ }^{17}$ J. A. Mejías, V. M. Jiménez, G. Lassaletta, A. Fernández, and J. P. Espinós, J. Phys. Chem. 100, 16255 (1996).

${ }^{18}$ R. Reiche, D. Dobler, J. P. Holgado, A. Barranco, A. I. MartínConcepción, F. Yubero, J. P. Espinós, and A. R. González-Elipe, Surf. Sci. 537, 228 (2003).

${ }^{19}$ J. Morasch, S. Li, J. Brötz, W. Jaegermann, and A. Klein, Phys. Status Solidi A 211, 93 (2014).

${ }^{20}$ J. Robertson, Eur. Phys. J. Appl. Phys. 28, 265 (2004).

${ }^{21}$ P. Dawson, M. Hargreave, and G. Wilkinson, J. Phys. Chem. Solids 34, 2201 (1973).

${ }^{22}$ J. P. Espinós, J. Morales, A. Barranco, A. Caballero, J. P. Holgado, and A. R. González-Elipe, J. Phys. Chem. B 106, 6921 (2002).

${ }^{23}$ J. Morales, J. P. Espinos, A. Caballero, A. R. Gonzalez-Elipe, and J. A. Mejias, J. Phys. Chem. B 109, 7758 (2005).

This article may be downloaded for personal use only. Any other use requires prior permission of the author and AIP Publishing. This article appeared in Appl. Phys. Lett. 110, 051603 (2017) and may be found at https://doi.org/10.1063/1.4975644.

Available under only the rights of use according to UrhG. 